\title{
Mielinolisis central pontina en trasplante hepático
}

\author{
LUIS CARTIER R., JOSÉ ARMIJO M., \\ GONZALO QUIROZ Z., JOSÉ MANUEL MATAMALA C.
}

Departamento de Ciencias Neurológicas Oriente, Facultad de Medicina, Universidad de Chile. Servicio de Neurología, Hospital del Salvador.

Recibido el 28 de diciembre de 2009, aceptado el 27 de septiembre de 2010 .

Correspondencia a: Dr. Luis Cartier R. E-mail: Icartier@med.uchile.cl

\section{Central pontine myelinolysis after liver transplantation. Report of five cases}

Background: Central Pontine Myelinolysis (CPM) is the most severe neurological complication after liver transplantation and apparently is not related to changes in osmolarity. Aim: To report five transplanted patients with CPM. Patients and Methods: In a series of 27 patients subjected to liver transplantation between 2005 and 2008, we found five patients who developed CPM. Results: All patients presented a severe hepatic encephalopathy. In the absence of alterations in osmolality, they developed, between the second to seventh day after transplantation, a central quadriplegia, hyperreflexia and Babinski sign, with preservation of sensorium. Magnetic resonance imaging showed demyelination of the motor pathway only in the protuberance. Motor recovery first began in the fingers and hands, followed by forearms, toes, feet, arms and finally the legs, defining a somatotopic recovery of the cortico-spinal pathway. Conclusions: This form of regaining motility shows that the selective involvement of the pyramidal tract in CPM, is according to its location in the pons and suggests a local entrapment. It is due to the structural rigidity of the protuberance that limits the expansive requirements of cytotoxic and vasogenic edema, and only affects the long fibers of cortico-spinal tracts, sensitized by encephalopathy. The entrapment syndromes generally preserve the axons, injure myelin and have no inflammatory reactions.

(Rev Med Chile 2010; 138: 1264-1271).

Key words: Encephalopathy, hepatic; Liver transplantation; Myelinolysis, central.
A dams y Víctor, el año 1959 publicaron cuatro casos de una nueva enfermedad, que denominaron "Mielinolisis central pontina” (MCP). Nombre que definía localización y contexto neuropatológico de este síndrome, atribuyendo su patogenia a un daño tóxico-nutricional. La histopatología reveló una desmielinización en el pie de la protuberancia, en ausencia de inflamación, daño neuronal o axonal ${ }^{1}$.

La MCP se expresa por un síndrome motor agudo, que daña el largo haz cortico-espinal a nivel protuberancial, generando paresia o plejia de las extremidades y eventualmente un síndrome de enclaustramiento. La resonancia magnética (RM) muestra imágenes localizadas en el puente, que tienen un aspecto triangular, como "tridente", o como "alas de murciélago", son hiperintensas en T2, y no captan contraste ${ }^{2}$. La RM puede no identificar la MCP en los primeros días, lo que no invalida su diagnóstico clínico ${ }^{3}$.

El clásico vínculo de la MCP con el alcoholismo ha hecho pensar que existen factores patogénicos comunes con otras enfermedades relacionadas con el alcohol, como la enfermedad de MarchiafavaBignami, caracterizada por la desmielinización del cuerpo calloso; o con el síndrome de Wernicke, que 
puede acompañarse de lesiones pontinas en 30\%, además de las clásicas lesiones del mesencéfalo y tubérculos mamilares ${ }^{4}$.

También se ha postulado como causa de la MCP una rápida e inadecuada corrección de la hiponatremia ${ }^{5}$. Stern y cols reportaron complicaciones neurológicas en pacientes con hiponatremia normalizada velozmente ${ }^{6,7}$. Otros autores, han sostenido que la causa de la mielinolisis no sería la rápida corrección de la hiponatremia, sino la brusca elevación del sodio sérico con esa maniobra $^{8}$. Conceptualizando así, la posible existencia de un "síndrome de desmielinización osmótica" . En el mismo sentido se pensó que debido al efecto inhibitorio del alcohol sobre la hormona antidiurética $(\mathrm{ADH})$, los alcohólicos podrían generar hiponatremia crónica, aunque resulta evidente que la desnutrición y el daño hepático son los factores más significativos en ellos. Se han comunicado numerosos casos de MCP no relacionados con hiponatremias corregidas, comenzando por los casos de Adams y Víctor ${ }^{1,10,11}$. Los casos de MCP reportados en trasplantes hepáticos, se producen en la etapa post-quirúrgica inmediata ${ }^{9,12}$ complicación no observada en trasplantes renales, cardíacos o pulmonares. Resultando la MCP la complicación neurológica más grave en los trasplantes hepáticos y causa de mortalidad en estos pacientes $^{12,13}$. La MCP puede representar hasta $17,2 \%$ de las complicaciones neurológicas en los trasplantados hepáticos ${ }^{14}$. Starzl fue el primero en relacionar la MCP con el trasplante hepático ${ }^{15}$.

Algunos investigadores aceptan que la MCP podría incluir lesiones parenquimatosas que comprometen otras áreas del encéfalo. Este daño fuera de la protuberancia fue denominado "mielinolisis extrapontinas" (MEP), intentando ampliar el concepto del clásico síndrome ${ }^{16}$.

Se describen cinco pacientes que después de un trasplante hepático se complicaron con un síndrome de mielinolisis central pontina no vinculado a correcciones electrolíticas.

\section{Pacientes y Métodos}

Se analizaron cinco pacientes que desarrollaron una mielinolisis central pontina en las etapas inmediatas al trasplante, de entre 27 sujetos que requirieron trasplante hepático entre los años 2005 y 2008 en el Hospital del Salvador. Los 27 enfermos recibieron una evaluación neurológica pre y post cirugía. Se hizo seguimiento específico sólo en aquellos pacientes que desarrollaron MCP.

\section{Caso 1}

Mujer de 36 años que cursaba embarazo de término, presentó ictericia, con bilirrubina de 7,8 mg/dl, enzimas (FA 1027; GOT 1002; GPT 747 ), protrombina $73 \%$ y ecografía abdominal normal. Dos días después tiene parto por vía vaginal. Al mes se detectó una bilirrubina total de $15,3 \mathrm{mg} / \mathrm{dl}$, protrombina de $11 \%$ y FA 364 , GOT y GPT en descenso. Se asoció dolor abdominal, vómitos y compromiso de conciencia. Ingresó con marcadores de Hepatitis B-C e IgM virus A, negativos. Tomografía cerebral (TC) normal. Estaba en coma, pupilas iguales reactivas, vagabundeo ocular, reflejos osteotendinos vivos, plantares indiferentes. Hipotensa (80/40), taquicárdica $\left(130 x^{\prime}\right)$, polipneica $\left(40 x^{\prime}\right)$, con mala perfusión distal. Se diagnosticó daño hepático fulminante, sepsis, neumonía y encefalopatía grado IV. Es trasplantada al tercer día de su ingreso, en equilibrio electrolítico. Al día siguiente, somnolienta, abría los ojos, movilizaba las cuatro extremidades con reflejos vivos y Babinski. Se mantuvo despierta y activa. Al séptimo día protrombina de $70 \%$, conciente, orientada, déficit motor de las cuatro extremidades, reflejos exaltados en extremidades inferiores y Babinski. En los días subsiguientes mejoró la comunicación verbal, inició recuperación motora en los dedos de las manos. Mantenía hiperreflexia, sin compromiso sensitivo. La RM del vigésimo día, mostró lesión en la región central de la protuberancia, visible en T2 y FLAIR, y lesiones de la corteza cingular, de aspecto hipóxico. El EEG tenía actividad lenta inespecífica. Completó la recuperación motora en dos meses, época en que inició rechazo del trasplante, definido por biopsia. Se instala infección respiratoria aguda. Al tercer mes, no hablaba por la traqueostomía, temblor postural de manos y pies, sin déficit motor, reflejos osteotendinos simétricos, plantares indiferentes. Posteriormente, se detectaron abscesos hepáticos múltiples. Es retrasplantada con examen neurológico y EEG normal. Desarrolló nueva infección pulmonar con flora multiresistente y shock séptico. Se encuentra ascitis, y trombosis de la vena cava, que la llevan al colapso hemodinámico. Fallece 120 días después del segundo trasplante. 


\section{Caso 2}

Mujer de 53 años sin antecedentes de alcoholismo, que cursaba con ictericia y baja de peso desde hacía un año. Fue estudiada con diagnóstico de hepatitis autoinmune $\mathrm{v} / \mathrm{s}$ cirrosis biliar primaria. Destacó AML (+), AAM (+), GGT y FA muy elevadas y varices esofágicas. Recibió tratamiento esteroidal, que luego se asoció azatioprina, sin lograr mejoría. Ingresó ictérica con múltiples equímosis, hipotensa, con diagnóstico de daño hepático de etiología no precisada, hipertensión portal y encefalopatía hepática grado III. Tenía bilirrubina total $31,4 \mathrm{mg} / \mathrm{dl}$, esplenomegalia, trombocitopenia y falla renal, sin cambios electrolíticos significativos, VHB y VHC (-). Soporosa, movilizaba sus extremidades, EEG lento encefalopático, TC cerebral mostraba edema significativo. Seis días después, en sopor profundo con encefalopatía grado IV es trasplantada. Al segundo día adquiría vigilancia aunque persistía somnolienta, dirigía la mirada tenía pupilas isocóricas reactivas. Al séptimo día se mantenía somnolienta, hipotónica, cuadripléjica, reflejos presentes y Babinski, sin alteraciones sensitivas. TC cerebral sin lesiones. Se mantuvo sin cambios. Se realizó RM a los quince días del trasplante, mostrando alteraciones en la protuberancia, hipointensas en $\mathrm{T} 1$ e hiperintensas en T2. El EEG encefalopático, tenía frecuencias theta, de mediano voltaje. Al trigésimo tercer día no había logrado recuperación motora, se mantenía soporosa. Se repitió la RM que mostró ampliación de la lesión protuberanciales (Figura 1). Falleció por complicaciones respiratorias al cuadragésimo día del trasplante.

\section{Caso 3}

Mujer de 62 años diabética, portadora de granulomas anulares, controlados en dermatología. Desde hacía un mes, tenía nauseas, diarreas frecuentes, ictericia, coluria, acolia y baja de peso. Enzimas hepáticas (GPT 2003, GOT 2670, FA 232, BT 20.2,), protrombina 27\%, VHB - C negativos. Ecografía abdominal mostró hígado disminuido de tamaño y colelitiasis confirmada por TC abdominal, con signos de hepatopatía crónica. Paciente soporosa, poco reactiva. Se diagnosticó hepatitis fulminante de causa no precisada, sobre daño hepático crónico. Continuó grave, inconsciente, respuestas de descerebración, pupilas reactivas, reflejos oculocefálicos presentes, reflejos osteotendineos abolidos, Babinski bilateral. La TC cerebral mostró ventrículos pequeños y edema cerebral. Se instaló captor de PIC. Es trasplantada con encefalopatía hepática grado IV sin alteraciones osmolares. A las 24 horas coma reactivo, flacidez de extremidades, arreflexia, plantares indiferentes, pupilas isocóricas reactivas. Día tercero, ojos abiertos, no dirige la mirada, pestañea a la amenaza, cuadriplejia arrefléctica. EEG encefalopático. Al quinto día vigil, dirigía la mirada, cuadriplejia arrefléctica, Babinski. Al octavo día protrombina de $100 \%$. Estaba activa, cumplía órdenes, no se expresaba por intubación, movía con facilidad la cabeza y distalmente las extremidades superiores, mantenía plejia de extremidades inferiores. Extubada, noveno día, vigil, activa, lenguaje conservado, cuadriparética, sin trastorno sensitivo. Día catorce lenguaje normal, cuadriparética proximal, mueve ambas manos y realiza prehensión, flexta y extiende antebrazos, no eleva los brazos, aunque eleva los hombros, logra flexión plantar, no hay flexión de rodillas, arreflexia y Babinski. La RM de día 22, mostró cambios de señal en protuberancia, visibles en la secuencia T2 y FLAIR (Figuras 2 y 3 ). La difusión y el mapa ADC mostraron alteración a ese nivel. La EMG definió neuropatía crónica

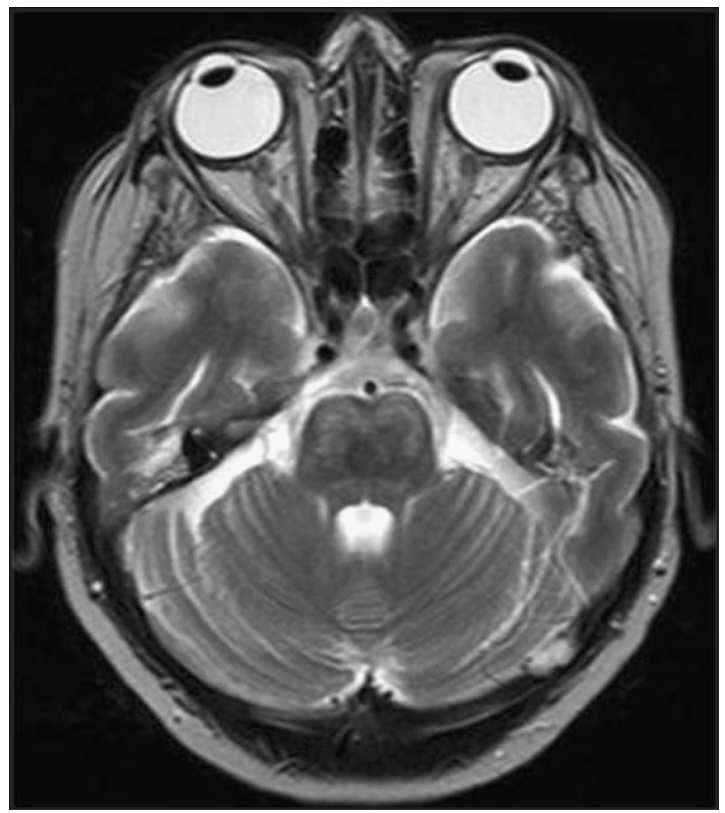

Figura 1. Resonancia Magnética de la paciente $N^{\circ} 2$, después de 33 días post trasplante. La imagen en corte axial de secuencia T2 muestra una hiperintensidad del pie de la protuberancia, que toma la apariencia de alas de murciélago. 


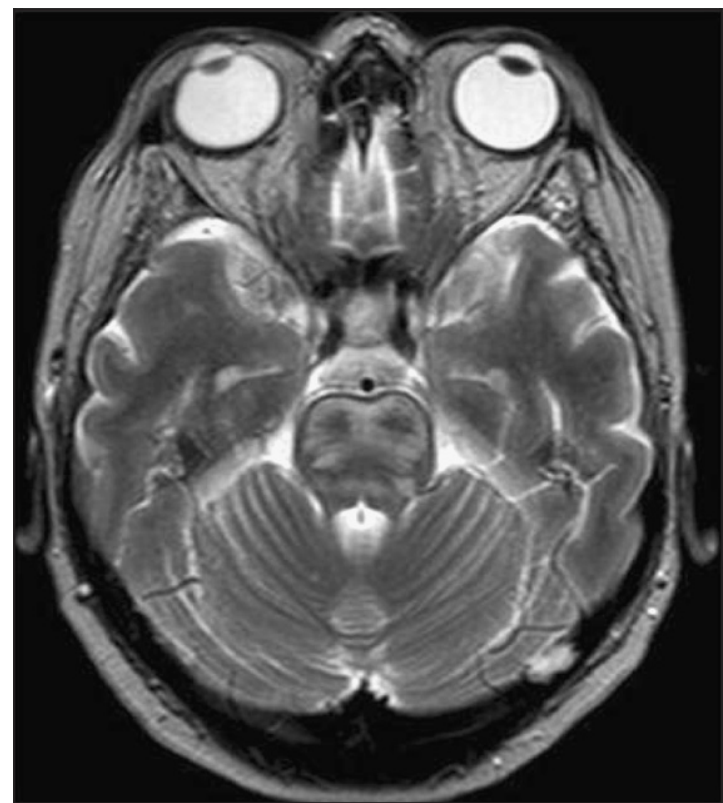

Figura 2. Resonancia Magnética de la paciente $N^{\circ} 3$, después de 30 días post trasplante. La imagen en corte axial de secuencia T2 muestra una hiperintensidad que abarca prácticamente todo el pie de la protuberancia, que adquiere la imagen de un antifaz.

que explicaría la arreflexia. Estando en buenas condiciones, se produce brusca hipotensión, pérdida de conciencia y coma que duró varios días, con reaparición de la cuadriparesia. La nueva RM definió lesiones corticales isquémicas y la lesión protuberancial. Dada de alta un mes después, cuadriparética y deterioro cognitivo.

\section{Caso 4}

Mujer de 45 años, inició compromiso de conciencia progresivo 24 horas antes de ingresar al Servicio de Urgencia. Tenía antecedentes de hepatitis medicamentosa cinco años antes (ibuprofeno). Estaba ictérica, bilirrubina total $26,5 \mathrm{mg} / \mathrm{dl}$, VHB-A-C (-), protrombina 69\%, comprometida de conciencia. Se diagnosticó hepatitis fulminante de etiología no establecida y encefalopatía grado III. No adquiría vigilancia, pupilas isocóricas reactivas, movía sus extremidades. TC cerebral con edema moderado. Dos días después protrombina de 19\%, en días subsiguientes 11\%. Es Trasplante con una encefalopatía grado IV al sexto día, en equilibrio electrolítico. Al día siguiente adquiría vigilancia, pestañaba a la amenaza, pupilas mió-

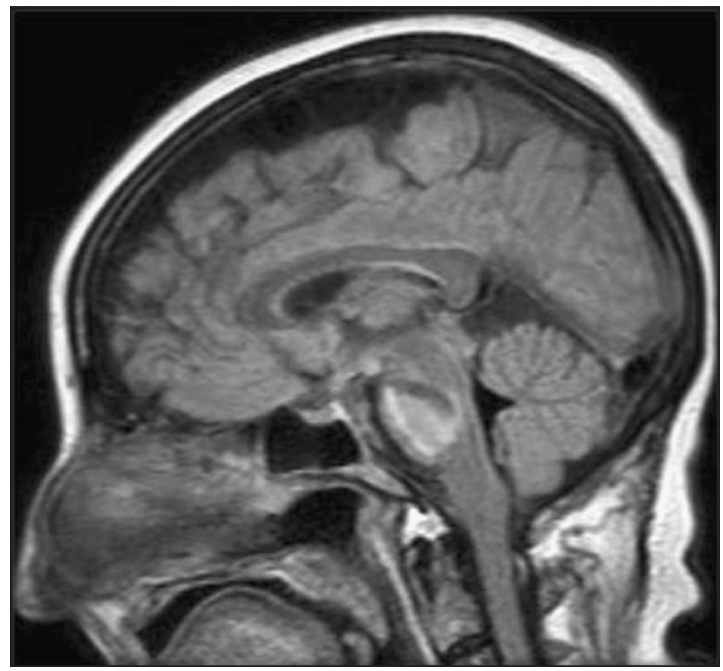

Figura 3. Resonancia Magnética de la paciente $N^{\circ} 3$, después de 30 días post trasplante. La imagen en corte sagital de secuencia FLAIR muestra una extensa hiperintensidad que abarca todo el pie de la protuberancia tomando una forma almendrada.

ticas reactivas, pero no movía las extremidades, reflejos vivos, Babinski. Al tercer día vigil, tetraplejia, hiperrefléctica. Habla al cuarto día, todavía confusa, ha comenzado a mover las manos. Al quinto día se inicia verborrea y delirio, moviliza algo más la mano izquierda que la derecha. Al día siguiente se intensifica cuadro sicótico, mueve con mayor potencia las manos, logra sostener el antebrazo izquierdo y se esbozan movimientos de pies. Séptimo día post-trasplante tranquila, inatenta, cuadriparética, sin movilidad proximal de brazos ni piernas. Al duodécimo día mantiene cuadriparesia proximal, hiperreflexia y Babinski, sin trastorno sensitivo. Se realiza electromiografía que descarta compromiso periférico y RM que no muestra lesión del puente. Continúa mejorando y al mes se va recuperada.

\section{Caso 5}

Mujer de 36 años, se quejaba de diarrea y baja de peso desde hacía seis meses. Hacía dos, asoció hiperbilirrubinemia de 2,48, protrombina $28 \%$, transaminasas sobre 2.000, Ecografía abdominal demostró hígado graso y colelitiasis, VHA-B-C negativos. Anticuerpos antimitocondriales $y$ antimúsculo liso, negativos. ANA (+) 1/320. TC cerebral normal. Encefalopatía grado II/III estable 
hemodinámicamente, sin falla renal, bilirrubina $20,5 \mathrm{mg} / \mathrm{dl}$. Somnolienta, lenguaje conservado, moviliza las extremidades, reflejos vivos, plantares flexores, sin trastornos sensitivos. Tres días después coma mínimamente reactivo, sin déficit motor central. Insuficiencia hepática subaguda, secundaria a hepatitis fulminante. En encefalopatía grado IV se realizó trasplante hepático sin alteraciones electrolíticas. Al día siguiente muy somnolienta, adquiría vigilancia, reflejos osteotendinos presentes. Al quinto día conciente orientada, cuadriparesia de predominio proximal, capaz de mover manos y dedos, con potencia muscular disminuida, incapaz de flectar o elevar los brazos, mueve los dedos de los pies. Hiperreflexia generalizada. Al séptimo día comprometida de conciencia, cuadripléjica hiperrefléctica con Babinski. EEG lento encefalopático. La RM mostraba hipointensidad en Tlen la región motora del puente e hiperintensa en T2, diseñando alas de murciélago (Figura 4). Del día decimoquinto en adelante, vigil, no dirige la mirada, cuadripléjica. Queda en estado vegetativo persistente.

\section{Resultados}

De los cinco enfermos descritos, ninguno presentó alteraciones del sodio que requiriera una corrección activa. Este grupo tampoco tenía antecedentes de abuso de alcohol, ni mostraban evidencias de un estado carencial. Su condición común era el daño hepático agudo, asociado a grave encefalopatía (Tabla 1). El compromiso motor aislado, sin alteraciones sensitivas, de pares craneanos, ni cerebelosas se instaló después del acto quirúrgico. La evolución del compromiso motor fue favorable mayoritariamente (3/5), concordante con un daño que sólo afecta la mielina (desmielinizante) (Tabla 2). El desenlace dependió de variados factores, en cada paciente. Tres pacientes mostraron lesiones extrapontinas en la RM, cuya naturaleza era independiente del fenómeno desmielinizante del puente y atribuible a hipotensión o hipoxia, concomitante a las contingencias clínicas de cada enfermo.

\section{Comentario}

Hemos analizado cinco pacientes que se complicaron con mielinolisis central pontina, después

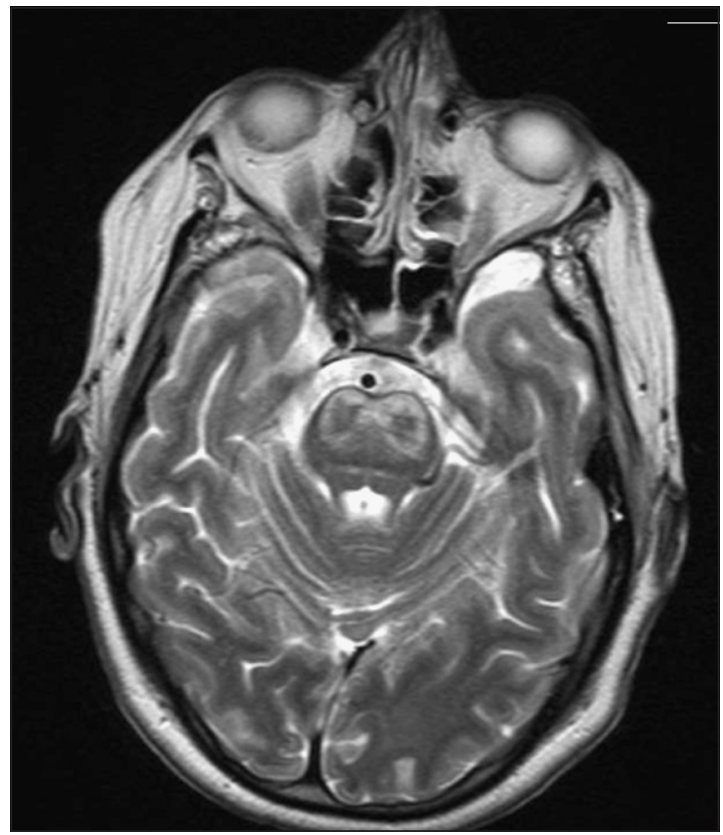

Figura 4. Resonancia Magnética de la paciente № 5 , a los 15 días del trasplante. La imagen en corte axial de secuencia T2 muestra una amplia hiperintensidad que compromete casi completamente el pie de la protuberancia.

de sendos trasplantes hepáticos, que evolucionaron sin alteraciones de la natremia. Las complicaciones neurológicas en los trasplantes hepáticos son frecuentes y atribuibles a diferentes causas ${ }^{10-13}$. En un análisis retrospectivo de 142 pacientes con trasplantes hepáticos Yu y cols ${ }^{10}$ encontraron complicaciones neurológicas en el 9,2\% y desarrollo de MCP en ocho, 5,6\%. Mellado y cols ${ }^{14}$ en 76 trasplantados encontraron $30,3 \%$ de complicaciones neurológicas y cuatro MCP, 5,2\%.

El daño neurológico de origen hepático ${ }^{17,18}$, así como los síndromes disosmolares, con los que se ha querido identificar patogénicamente la $\mathrm{MCP}^{9}$, actúan en todo el encéfalo, excediendo ampliamente la protuberancia. Por ello, sorprende que las largas fibras motoras se lesionen únicamente a nivel del puente, surgiendo la idea de un factor local, además de los generales ${ }^{19}$.

Creemos que la protuberancia se comporta rígidamente ante los requerimientos expansivos que originan los edemas citotóxicos o vasogénicos. Debido a que posee una configuración inextensible, producto de los miles de fibras dispuestas entre 

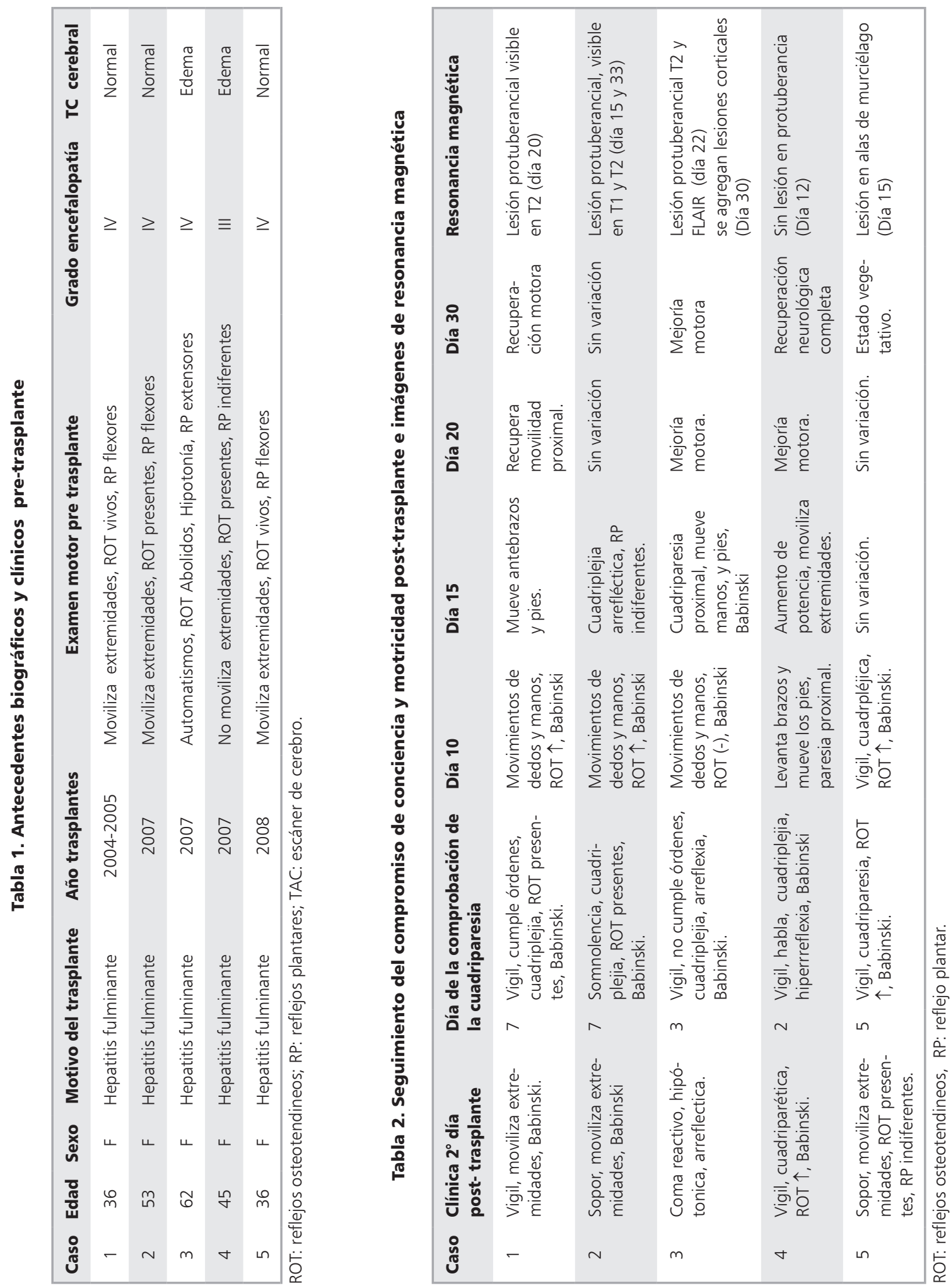
ambos hemisferios cerebelosos que envuelven el contorno del puente (comisura cerebelosa). Reforzadas por una malla interna, constituida por el mayor contingente de fibras que llega al puente, que cruza transversalmente la protuberancia desde los núcleos pontinos al hemisferio cerebeloso contralateral (haz cortico-ponto-cerebeloso) y un rafe central rígido. Situación que crean una condición que es única en el neuroeje, atrapando los haces cortico-espinales en las áreas anterolaterales del puente, donde pueden ser comprimidos. El haz piramidal por el grosor y longitud de sus fibras es el más vulnerable del SNC. Por ello las alteraciones biomoleculares adversas que genera la encefalopatía, asociadas a los posibles efectos deletéreos que origina el hígado hipóxico del trasplante ${ }^{20}$ completan la sensibilización y vulnerabilidad de la vía motora. Cualquier síndrome por atrapamiento, destruye la mielina en las áreas comprimidas, sin originar reacciones inflamatorias, como fue la clásica descripción de la MCP.

El compromiso motor de estos pacientes fue de predominio proximal en intensidad y tiempo. La recuperación se inició por los dedos y las manos, luego siguieron antebrazos y brazos, posteriormente los ortejos, pies y finalmente las piernas. Poniendo en evidencia una somatotopía en la recuperación de las fibras motoras, que da significado a este preferente y selectivo compromiso, concordante con las imágenes de la $\mathrm{RM}^{21,22}$.

Pensamos que las denominadas lesiones extrapontinas no concuerdan con las hipótesis patogénicas postuladas para la $\mathrm{MCP}^{9,23,24}$. Además, resulta difícil establecer correlaciones etiopatogénicas sólo con imágenes de RM, que muestran lesiones unas veces en la corteza cerebral ${ }^{25}$, otras en la substancia blanca subcortical ${ }^{26}$, en el cerebelo ${ }^{27}$, el tálamo ${ }^{24}$, el putamen o los núcleos caudados ${ }^{28,}$ ${ }^{29}$. La constante variación en la ubicación y características de las imágenes extrapontinas, les hacen perder validez etiopatogénica, especialmente al compararlas con las lesiones del puente que son similares en todas las MCPs.

También el término "desmielinización osmótica" parece inadecuado para rebautizar a la "mielinolisis central pontina". Porque carece de realidad patogénica, e implicaría aceptar que la pérdida de la mielina de los axones motores, sólo en el puente, tiene su origen en los cambios de la concentración osmolar de los electrolitos plasmáticos, como única causa.
Este es un estudio original que nos permitió postular y validar desde la clínica la hipótesis mecánica de la MCP y explicar la condición exclusiva de la protuberancia para poder sufrir estas lesiones. Creemos haber hecho comprensible el significado $\mathrm{y}$ alcance de la mielinosis del puente.

Agradecimientos. Los autores agradecen al equipo de Trasplante Hepático del Hospital del Salvador que nos permitieron seguir a sus pacientes durante largos períodos. Agradecemos la gentileza y desinterés de los doctores Waldo Martínez M., Mario Uribe M., Felipe Catan G., Rodrigo Zapata L. y a la E.U. Ana María Basáez L. por su inestimable colaboración.

\section{Referencias}

1. Adams RA, Víctor M, Mancall EL. Central pontine myelinolysis: a hitherto undescribed disease occurring in alcoholics and malnourished patients. Archives of Neurology Psychiatry 1959; 81: 154-72.

2. Guo Y, Hu JH, Lin W, Zheng KH. Central pontine myelinolysis after liver transplantation: MR diffusion, spectroscopy and perfusion findings. Magnetic Resonance Imaging 2006; 24: 1395-8.

3. Miller GM, Baker Jr HL, Olkazaki H, Whisnant JP. Central pontine myelinolysis and its imitators: MR findings. Radiology 1988; 168: 795-802.

4. Víctor M, Laureno R. Neurologic complications of alcohol abuse: epidemiologic aspects. Adv Neurol 1978; 19: 603-17.

5. Laureno R, Illowsky B. Myelinolysis after Correction of Hyponatremia. Ann Inter Med 1997; 126: 57-62.

6. Sterns RH, Capuccio JD, Silver SM, Cohen EP. Neurologic sequelae after treatment of severe hyponatrenia: a multicenter perspective. J Am Soc Nephrol 1994; 4: 1522-33.

7. Sterns R, Silver SM. Brain volume regulation in response to hypo-osmolality and correction. The American Journal of Medicine 2006; 119: S12-6.

8. Verbalis JG, Gullans SR. Rapid correction of hyponatremia produces differential effects on brain osmolyte and electrolyte reaccumulation in rats. Brain Res 1993; 606: 19-27.

9. Sterns RH, Riggs JE, Schochet SS. Osmotic demyelination syndrome following correction of hyponatremia. $\mathrm{N}$ Engl Med 1986; 314: 1535-42.

10. Yu J, Zheng SS, Liang TB, Shen Y, Wang WL, Ke QH. Possible cause of central pontine myelinolysis after liver 
transplantation. World J Gastroenterol 2004; 10: 2540-3.

11. Mast H, Gordon PH, Mohr JP, Tatemichi TK. Central Pontine myelinolysis: clinical syndrome with normal serum sodium. Eur J Med Res 1995; 1: 168-70.

12. Saner FH, Sotiropoulos GC, Gu Y, Paul A, Radtke A, Gensicke J, et al. Severe neurological events following liver transplantation. Arch Med Res 2007; 38: 75-9.

13. Lewis MB, Howdle PD. Neurologic complications of liver transplantation in adults. Neurology 2003; 61: 1174-8.

14. Mellado P, Peredo P, Valenzuela R, Arrese M, Pérez RM, Domínguez P, et al. Neurological complications following a liver transplantation: experience from a Chilean center. Rev Med Chile 2008; 136: 1255-63.

15. Starzl TE, Schneck SA, Mazzoni G, Aldrete JA, Porter KA, Schröter GP, et al. Acute neurological complications after liver transplantation with particular reference to introperative cerebral air embolus. Ann Surg 1978; 187: 236-40.

16. Wright DG, Laureno R, Victor M. Pontine and extrapontine myelinolysis. Brain 1979; 102: 36-85.

17. Weissenborn K, Bokemeyer M, Krause J, Ennen J, Ahl B. Neurological and neuropsychiatric syndromes associated with liver disease. AIDS 2005; 19: S93-8.

18. Saner FH, Gu Y, Minouchehr S, Ilker K, Fruhauf N, Paul A, et al. Neurological complications after cadaveric and living donor liver transplantation. J Neurol 2006; 253: 612-7.

19. Arieff AI, Ayus JC. Treatment of symptomatic hyponatremia: Neither haste nor waste. Crit Care Med 1991; 19: 748-51.

20. Romanque P, Uribe M, Videla LA., Molecular mecha- nisms in liver ischemic-reperfusion injury and ischemic preconditioning. Rev Med Chile 2005; 133: 469-76.

21. Guo Y, Hu JH, Lin W, Zheng KH. Central pontine myelinolysis after liver transplantation: MR diffusion, spectroscopy and perfusion findings. Magnetic Resonance Imaging 2006; 24: 1395-8.

22. Ruzek K, Campeau N, Miller G. Early diagnosis of central pontina myelinolysis with diffusion-weighted imaging. AJNR Am J Neuroradiol 2004; 25: 210-3.

23. Lawson B, Silva J. Mielinolisis central pontina e hiponatremia. Un problema no resuelto. Rev Med Chile 2001; 129: 427-32.

24. Abbott R, Silber E, Felber J, Ekpo E. Osmotic demyelination syndrome. BMJ 2005; 331: 829-30.

25. Colakos N, Fischbein N, Baringer JR, Jay CH. Cortical MRI findings associated with rapid correction of hiponatremia. Neurology 2000; 55: 1048-51.

26. Bourgouin P, Chalk C, Richardson $\mathrm{CH}$, Duang H, Vezina J. Subcortical White Matter Lesions in Osmotic Demyelination Syndrome. AJNR Am J Neuroradiol 1995; 16: 1495-7.

27. Huq S, Wong M, Chan H, Crimmins D. Osmotic demyelination syndromes: Central and extrapontine mielinolisis. J Clin Neurosci 2007; 14: 684-8.

28. Seah AB, Chan LL, Wong MC, Tan EK. Evolving spectrum of movement disorders in extrapontin and central pontina myelinolysis. parkinsonisnm and related disorders 2002, 9, 117-9.

29. Seok JI, Youn J, Chung EJ, Lee WY. Sequential observation of movement disorders and brain images in the case of central pontine myelinolysis and extrapontine myelinolysis. Parkinsonism Relat Disord 2006; 12: 462-4. 